\section{Putting on the brakes}

\section{By Tracey Baas, Senior Editor}

The key safety concern with chimeric antigen receptors is their inability to distinguish whether healthy or malignant cells are expressing a target antigen. A group at the Memorial Sloan-Kettering Cancer Center thinks it has solved the problem with T cells expressing two antigenspecific receptors to distinguish on-target cancer cells from off-target normal cells. ${ }^{1}$

Chimeric antigen receptor (CAR) T cells currently in the clinic are made up of an antigen-specific single-chain variable fragment ( $\mathrm{scFv}$ ) fused to T cell-activating and-co-stimulatory domains. The CAR is designed to bind to an extracellular, tumor-associated antigen, and the stimulatory domains cause $\mathrm{T}$ cells to activate and proliferate, thus eliminating tumor cells.

The MSKCC team went a step further by including in their T cellbased therapeutics another CAR-an inhibitory CAR (iCAR) that fused an $\mathrm{scFv}$ to a $\mathrm{T}$ cell inhibitory signaling domain (see Figure 1, "Putting the brakes or gas on T cell function").

As proof of concept, the group focused on two antigens: CD19 and prostate-specific membrane antigen (PSMA; FOLH1; GCPII). CD19 is overexpressed on malignant $\mathrm{B}$ cells but also is found on normal B cells. PSMA is overexpressed on metastatic prostate cancers but also is found on normal kidney, liver and colon cells, and brain astrocytes.

In the standard CAR, the researchers used T cell-signaling domains from $\mathrm{CD} 3 \zeta$ and $\mathrm{CD} 28$ to activate and amplify $\mathrm{T}$ cell function. Both domains are common components of most CARs in the clinic.

For the new iCAR, the group used the T cell inhibitory domain from programmed cell death 1 (PDCD1; PD-1; CD279). PD-1 is an immune checkpoint receptor that can impede $T$ cell antitumor immunity when bound by tumor-expressed programmed cell death 1 ligand 1 (CD274 molecule; PD-L1; B7-H1). Companies are pursuing PD-1 itself as a target. CureTech Ltd., Merck \& Co. Inc. and Ono Pharmaceutical Co. Ltd. each have anti-PD-1 mAbs in clinical trials.

The team tested its iCAR on T cells engineered to express either a conventional CAR or an endogenous receptor for antigen called a $\mathrm{T}$ cell receptor (TCR).

The results were striking-both in vitro and in mice. In all cases, $\mathrm{T}$ cells expressing an iCAR plus a CAR or TCR sought out and destroyed on-target cells. Off-target cells were protected.

For example, a fibroblast-reactive TCR plus the PSMA-specific iCAR was active against PSMA- fibroblast cultures and showed fibroblast killing ability and high levels of cytokine secretion. In PSMA ${ }^{+}$fibroblast cultures, the T cells expressing the same fibroblast-specific TCR plus the PSMA-specific iCAR showed decreased cytotoxicity and cytokine secretion compared with $\mathrm{T}$ cells expressing the fibroblast-specific TCR alone, suggesting that iCARs put the brakes on TCR-based T cell function.

Similar results were seen with T cells expressing a CD19-specific CAR plus the PSMA-specific iCAR in cell culture and mice. T cells with combined CAR and iCAR expression eliminated on-target $\mathrm{CD} 19^{+} / \mathrm{PSMA}^{-}$cells but spared $\mathrm{CD} 19^{+} / \mathrm{PSMA}^{+}$cells, indicating that iCARs also put the brakes on CAR-based $\mathrm{T}$ cell function.

The group also showed that the strategy could distinguish mixed on-target and off-target cells. To do so, the researchers made a coculture of green fluorescent, on-target $\mathrm{CD} 19^{+} / \mathrm{PSMA}^{-}$artificial antigenpresenting cells and red fluorescent, off-target CD $19^{+} / \mathrm{PSMA}^{+}$artificial antigen-presenting cells.

Time-lapse microscopy showed that green on-target cells and red off-target cells were eliminated at a similar rate by $\mathrm{T}$ cells expressing a CD19-specific CAR. In contrast, T cells expressing the CD19-specific CAR plus the PSMA-specific iCAR eliminated green cells and spared red cells. After 38 hours there was about an 85\% decrease in on-target cells and a $10 \%$ decrease in off-target cells.

Together, these results suggest that the iCAR $\mathrm{T}$ cell system can selectively inhibit $\mathrm{T}$ cells from engaging in off-target effects while still allowing them to eliminate on-target cells.

Results were published in Science Translational Medicine. The team was led by Michel Sadelain, director of MSKCC's Center for Cell Engineering.

"Few antigens are completely specific to tumor tissue, so broadening the applicability of CAR therapy will be dependent upon finding ways to target tumor antigens that are also expressed to a degree on healthy tissue," noted Michael Gladstone, an associate in the life sciences group at Atlas Venture. "This new work is an excellent indication that iCAR strategies may be a viable way to expand the tumor antigens that may safely be targeted with highly potent CAR therapy."

Stewart Abbot, executive director of integrative research at Celgene Cellular Therapeutics, a subsidiary of Celgene Corp., said that the strategy is an elegant first step to provide a molecularly encoded control for $\mathrm{T}$ cell-based therapeutics.

"The inclusion of inhibitory signaling into engineered $\mathrm{T}$ cells provides the possibility to limit potential normal tissue destruction without entirely ablating the therapeutic agent," he said.

\section{Map for the iCAR}

Sadelain told SciBX that his goal is to pursue iCARs in the solid tumor setting, which contrasts with conventional CARs that are in development for blood cancers.

"We are interested in first taking our system into solid tumors, where there are no targets with a profile as attractive as that of CD19," he said. "We will not apply the iCAR approach to the CD19 setting."

Sadelain declined to comment on specific solid tumor target combinations. 
Figure 1. Putting the brakes or gas on $\mathbf{T}$ cell function. Inhibitory chimeric antigen receptors (iCARs) put the brakes on T cell function upon encountering off-target cells. The iCAR is made up of an antigen-specific single-chain variable fragment (scFv) fused to a T cell inhibitory signaling domain. The CAR is made up of an antigen-specific scFV fused to a T cell-activating domain. Cells expressing a tumor-associated antigen (antigen A) but not a normal-tissue antigen (antigen B) would induce T cell activation (solid line), cytotoxicity and cytokine signaling to kill the on-target cells (I.1). In contrast, in cells expressing both antigen A and antigen $B$, the iCAR would inhibit CARinduced or T cell receptor (TCR)-induced T cell activation and prevent subsequent cytotoxicity and cytokine signaling to spare the off-target cells (I.2).

Co-stimulatory chimeric antigen receptors (CCRs) were used to ramp up T cell function when encountering on-target cells. The CAR or TCR used is designed to show low affinity for its antigen. In this scenario, cells expressing antigen $A$ (such as a tumor-associated antigen that might also be found on normal tissue) but not antigen B (such as a tumor-associated antigen) would only show suboptimal $\mathrm{T}$ cell activation

(dashed line) and lack cytotoxicity and cytokine signaling toward off-target cells (II.1). In cells expressing both antigen A and antigen B, the CCR would help to induce CAR-mediated or TCR-mediated T cell activation and result in cytotoxicity and cytokine signaling to eliminate the on-target cells (II.2)

Sadelain's laboratory was also part of a team that designed $\mathrm{T}$ cells expressing two different receptors that ramped up $\mathrm{T}$ cell function when encountering on-target cells while sparing off-target cells. Results were published early in 2013 (ref. 2; see Box 1, "Revving up T cells").

CD19-specific, CAR-based T cell therapies eliminate CD19expressing $\mathrm{B}$ cell malignancies and have shown dramatic results in the clinic. The side effect of elimination of healthy B cells is considered a reasonable trade-off even though some patients may need to undergo a lifetime of immunoglobulin replacement therapy.

Sadelain said that another application of the iCARs is in graft-versushost disease (GvHD). He said that Marcel van den Brink and his lab are working together to design iCARs to prevent GvHD during bone marrow transplant-the iCARs block the attack of donor immune cells on the recipient's normal tissue while allowing attack on the recipient's cancer cells.

van den Brink is the head of hematologic oncology at MSKCC and specializes in allogeneic blood stem cell transplantation for adult patients with cancer.

Regardless of indication, Gladstone said, "there are still limitations with identifying and selecting iCAR antigens that are reliably and sufficiently robustly expressed on normal tissues but not on tumor tissue. Another potential challenge is defining the CAR cell product."

For each antigen combination, absolute and relative levels of CAR and
iCAR expression on the T cells will have to be determined, as well as how stable the CAR and iCAR expression is in vivo over time.

"Tumor antigens can be quite heterogeneous between patients and even within a given patient_-both on tumor and on healthy tissue-so it is critical to understand whether variation of CAR and/or iCAR expression or antigen density disrupts the safety and efficacy margins exemplified in their study," said Gladstone.

Abbot saw at least two other potential limitations. "The current proof-of-concept studies are well conducted, but the iCAR targets may be expressed at higher levels than would be expected in normal tissues," he said. "The inhibitory potential of
"This new work is an excellent indication that iCAR strategies may be a viable way to expand the tumor antigens that may safely be targeted with highly potent CAR therapy."

-Michael Gladstone, Atlas Venture the iCARs when engaging physiologically relevant concentrations of their target antigens remains to be defined."

He added, "Additionally, the effect of chronic co-stimulation of iCAR and CAR remains to be tested. The authors themselves point out that there may be the possibility for some-arguably the majority-of the T cells expressing CAR plus iCAR to become energized or exhausted over time." 


\section{Box 1. Revving up T cells.}

Michel Sadelain, director of the Memorial Sloan-Kettering Cancer Center's Center for Cell Engineering, previously teamed with researchers from the Dresden University of Technology to design co-stimulatory chimeric receptors (CCRs) that ramp up T cell function when encountering on-target cells while sparing off-target cells. ${ }^{2}$ Similar to the newly reported inhibitory chimeric antigen receptors (iCARs), the goal is to have $T$ cells express two different receptors that eliminate cancer cells and spare normal cells (see Figure 1, "Putting the brakes or gas on T cell function").

"Having two approaches to achieve tumor selectivity in the absence of a tumor-unique target is very valuable, but both will require a balance of finely tuned signaling," said Sadelain.

In proof-of-concept studies, the MSKCC-Dresden group focused on prostate stem cell antigen (PSCA) and prostate-specific membrane antigen (PSMA; FOLH1; GCPII). Both targets are highly expressed in metastatic prostate cancer, although neither antigen is absolutely specific to prostate tissue.

PSCA also is expressed in the renal pelvis, ureter, urinary bladder and urethra. PSMA is expressed in type II astrocytes, the kidney proximal tubule and the intestinal brush border.

In mice with $\mathrm{PSCA}^{+} / \mathrm{PSMA}^{+}$, $\mathrm{PSCA}^{+} / \mathrm{PSMA}^{-}$and $\mathrm{PSCA}^{-} / \mathrm{PSMA}^{+}$tumors, $\mathrm{T}$ cells expressing an attenuated, PSCA-specific CAR and a PSMA-specific CCR eradicated the double-antigen tumors but not the single-antigen tumors. The findings confirmed that $T$ cell cytotoxicity took place in the presence of on-target tissues while sparing off-target tissues.

Dario Campana, a professor of pediatrics at the National University of Singapore, thought that the main application would be in solid tumors and the immediate next steps should be testing the $\mathrm{T}$ cell strategies in a setting that is a better proxy for actual malignancies. Specifically, he said, the group should focus

"The inclusion of inhibitory signaling into engineered T cells provides the possibility to limit potential normal tissue destruction without entirely ablating the therapeutic agent."

-Stewart Abbot,

Celgene Cellular Therapeutics on two targets that are differentially expressed on human cancer and normal cells.

"While this is a very elegant and successful demonstration of a proof-of-concept iCAR study, to my knowledge there are no such tumors that coexpress PSMA and CD19," said Campana. "The authors mention other targetable antigen combinations, but whether these are truly differentially expressed in a substantial number of tumors or if they can produce similar effects remains to be seen.”

MSKCC has filed for a patent covering the use of iCARs to protect normal tissues from off-target effects of allogeneic or autologous T cells. The IP has been optioned to Juno Therapeutics Inc., which could give the biotech a leg up over other CAR companies. Juno was formed to commercialize immunotherapeutic discoveries by scientists at three institutions: MSKCC, Fred Hutchinson Cancer Research Center and the Seattle Children's Research Institute.

Baas, T. SciBX 7(2); doi:10.1038/scibx.2014.40

Published online Jan. 16, 2014

\section{REFERENCES}

1. Fedorov, V.D. et al. Sci. Transl. Med.; published online Dec. 11, 2013; doi:10.1126/scitranslmed.3006597

Contact: Michel Sadelain, Memorial Sloan-Kettering Cancer Center, New York, N.Y.

e-mail: m-sadelain@ski.mskcc.org

2. Kloss, C.C. et al. Nat Biotechnol. 31, 71-75 (2013)

\section{COMPANIES AND INSTITUTIONS MENTIONED}

Atlas Venture, Cambridge, Mass.

Celgene Corp. (NASDAQ:CELG), Summit, N.J.

CureTech Ltd., Yavne, Israel

Dresden University of Technology, Dresden, Germany Fred Hutchinson Cancer Research Center, Seattle, Wash. Juno Therapeutics Inc., Seattle, Wash.

Memorial Sloan-Kettering Cancer Center, New York, N.Y. Merck \& Co. Inc. (NYSE:MRK), Whitehouse Station, N.J. National University of Singapore, Singapore

Ono Pharmaceutical Co. Ltd. (Tokyo:4528), Osaka, Japan Seattle Children's Research Institute, Seattle, Wash. 